\title{
Inhalt des LXVIII.Bandes.
}

\section{Kanonistische Abteilung XXXVII.}

Walter von Hörmann zu Hörbach t. Von Nikolaus Graß IX Albert M. Koeniger f. Von Theodor Gottlob . . . . . XV

Engel, Wilhelm, Die Stadt Würzburg und die Kurie . . 303

Felbinger, Alfred, Die Primatialprivilegien für Italien von Gregor VII. bis Innocenz III. (Pisa, Grado, Salerno)

Lehmann, Konstantin, Die Entstehung der Freiheitsstrafe in den Klöstern des heiligen Pachomius . . . . . .

Lentze, Hans, Die Rechtsform der Altarpfründen im mittelalterlichen Wien........... . . . . . . . •

Lindner, Dominikus, Die Inkorporation im Bistum Regensburg seit dem Konzil von Trient . . . . . . . . . 164

\section{Miszellen:}

Lübeck, Konrad, Der erste Diözesanbischof des Klosters Fulda . . . . . . . . . . . . . . . . . . . . . 360

Merzbacher, Friedrich, Die Hexenprozesse im Hochstift Bamberg . . . . . . . . . . . . . . . . . . 376

\section{Literatur:}

Adam, Alfred, Die Nassauische Union von 1817 . . . . 482 Besprochen von Johannes Heckel.

Barlow, Frank, Durham Jurisdictional Peculiars . . 431 Besprochen von Hans Erich Feine.

Bauerreiß, Romuald, Kirchengeschichte Bayerns I

Besprochen von Hermann Nottarp.

Baur, Johannes, Die Brixener Synoden (Anzeige) . . 501

Bornkamm, Heinrich, Die Staatsidee im Kulturkampf 492 Besprochen von Johannes Heckel.

Ebers, Godehard Jos., Grundriß des Kathol. Kirchen. rechts

Besprochen von Hans Erich Feine.

Engel, Wilhelm, Die Ratschronik der Stadt Würzburg 
Engel, Wilhelm, Die mittelalterlichen Seelbücher des Kollegiatstiftes St. Gumbert zu Ansbach . . . . . . Besprochen von Hermann Nottarp.

Engel, Wilhelm, Würzburger in spätma. Bruderschaften der Stadt Rom (Anzeige) . . . . . . . . . . . .

一, -, Das Würzburger Heiltum des späten Mittelalters (Anzeige) . . . . . . . . . . . . . . . . . . .

Erdmann, Karl Dietrich, Volkssouveränität und Kirche $(1789-91)$.

Besprochen von Friedrich Giese.

Feine, Hans Erich, Kirchliche Rechtsgeschichte I: Die katholische Kirche (Selbstanzeige) . . . . . . . . . 390

Festgabe Karl Schornbaum (Anzeige) . . . . . . . . . 508

Festschrift Karl Haff (Anzeige) . . . . . . . . . . . . 508

Fink, Karl August, Das Vatikanische Archiv, 2. verm. Aufl. (Anzeige). . . . . . . . . . . . . . . . . . 499

Goetting, Hans, Die Anfänge des Reichsstifts Gandersheim (Anzeige). . . . . . . . . . . . . . . 500

Gottlob, Theodor, Gerichts- und Kanzleiordnung des Bischofs Marquard von Konstanz (Anzeige) . . . . . 500

Graß, Franz, Pfarrei und Gemeinde im Spiegel der Weistümer Tirols

Besprochen von Karl Haff.

Hallinger, Kassius, Gorze-Kluny . .. . . . . . . . 404

Besprochen von Hans Erich Feine.

Hartwig, H., Widukind in Geschichte und Sage. . . Besprochen von Hermann Nottarp.

Heckel, Johannes, Initia iuris ecclesiastici Protestan. tium (Selbstanzeige) . . . . . . . . . . . . . .

-, - Melanchthon und das heutige Staatskirchenrecht (Selbstanzeige)

Hermelink, Heinrich, Geschichte der evang. Kirche in Württemberg von der Reformation bis zur Gegenwart Besprochen von Hans Erich Feine.

Just, Leo, Zur Entstehungsgeschichte des Febronius (Anzeige) . . . . . . . . . . . . . . . . . . . .

Kempf, Friedrich, Die Register Innocenz' III.

-, -, Regestum Innocentii III papae super negotio Romani imperii . . . . . . . . . . . . . . . . . .

Besprochen von Herbert Grundmann.

Klinkenberg, H. M., Grundprobleme kirchlicher Ordnung in den ersten fünf Jahrhunderten (Anzeige) . . . 
Kuttner, Stephan, Concilar Law in the making (Lyon 1274).

,-- , The scientific investigation of mediaeval canon law.

-, - The Barcelona Edition of St. Raymonds first treatise of Canon Law (Anzeigen) . . . . . . . . . . . . 498

Kuujo, E. O., Das Zehntwesen der Erzdiözese HamburgBremen . . . . . . . . . . . . . . . . . . . . .

Besprochen von Hermann Nottarp.

Langer, Ellinor, Die Geschichte des Adligen Damenstifts zu Innsbruck . . . . . . . . . . . . . . . . . .

Besprochen von Hans Lentze.

Maaß, Ferdinand, Der Josephinismus. Quellen zu seiner Geschichte in Österreich $1760-90$. . . . . . . . . 464

Besprochen von Hans Erich Feine.

Mayer, Matthias, Der Tiroler Anteil des Erzbistums Salzburg . . . . . . . . . . . . . . . . . . . 456

Besprochen von Hans Lentze.

Mayer, Theodor, Fürsten und Staat. . . . . . . . . 396

Besprochen von Wilhelm Weizsäcker.

Merk, Karl Josef, Das Brevier und der Säkularklerus Besprochen von Hermann Nottarp.

Meyer, Otto, Uraha Sacra (Anzeige) .

507

Österreichische Geschichtswissenschaft in Selbstdarstellun. gen hg. von Nikolaus GraB (Anzeige) . . . . . .

Österreichisches Archiv für Kirchenrecht 1. Jg. 1950 (Anzeige). . . . . . . . . . . . . . . . . . . . . . 505

de Pange, Jean, Le roi très chrétien . . . . . . . . 394 Besprochen von Percy Ernst Schramm.

Panzram, Bernhard, Kirchliche Rechtsgeschichte und wissenschaftliche Rechtsvergleichung (Anzeige) . .

Rösser, Ernst, Die Stellung der Laien nach dem kanonischen Recht (Anzeige) . . . . . . . . . . . . . . 507

Schmidt, Aloys, Zur Geschichte der älteren Universität Würzburg (Anzeige) . . . . . . . . . . . . . . .

Schreiber, Georg, Der erste Entwurf des Reichskonkor$\operatorname{dats}(1920 / 21)$ (Anzeige) . . . . . . . . . . . . .

Stamer, Ludwig, Kirchengeschichte der Pfalz, I. und II. Band . . . . . . . . . . . . . . . . . . . .

Besprochen von Friedrich Giese.

Stickler, Alphonsus, Historia Iuris Canonici I: Historia fontium . . . . . . . . . . . . . . . . . . . . 390

Besprochen von Hans Erich Feine. 
Tierny, Brian, A conciliar theory of the 13th century (Anzeige) . . . . . . . . . . . . . . . . . . . . 498

Tüchle, Hermann, Kirchengeschichte Schwabens . . 443 Besprochen von Hans Erich Feine.

Vincke, Johannes, Die Gegenwart im Spiegel der mittelalterlichen Rechtsentwicklung (Anzeige) . . . . . 506

Volk, Paulus, Fünfhundert Jahre Bursfelder Kongregation . . . . . . . . . . . . . . . . . . . . . . 462

Besprochen von Hans Erich Feine.

Anzeigen . . . . . . . . . . . . . . . . . . . 498

Eingänge . . . . . . . . . . . . . 507

Zur Chronik. . . . . . . . . . . . . . 508

Kommission zur Herausgabe der Werke Martin Luthers. 509

Monumenta Germaniae Historica . . . . . . . 510

Abkürzungs- und Siglenverzeichnis . . . . . . 513

Alphabetisches Verzeichnis der Mitarbeiter an diesem Bande:

Prof. Dr. Wilhelm Engel in Würzburg, S. 303.

Prof. Dr. Hans Erich Feine in Tübingen, S. 390, 404, 431, 443, 462, 465. 498.

Dr. phil. Alfred Felbinger z. Z. in Rom, Collegio Greco, S. 95.

Prof. Dr. Friedrich Giese in Frankfurt, S. 440, 470.

Prof. Dr. Theodor Gottlob in Freiburg i. Br., S.XV.

Prof. Dr. Dr. Nikolaus GraB in Innsbruck, S. IX.

Prof. Dr. Herbert Grundmann in Münster, S.416.

Prof. Dr. Karl Haff in Hamburg, S. 449.

Prof. D. Dr. Johannes Heckel in Feldkirchen bei München, S. 474, 478, 482, 492.

Staatsanwalt Dr. Konstantin Lehmann in Tübingen, S. 1.

Priv.-Doz. Dr. Han s Lentze, Innsbruck, Stift Wilten, S. 221, 456, 460.

Prof. Dr. Dominikus Lindner in Freising, S. 164.

Prof. Dr. Konrad Lübeck in Fulda, S. 360.

Dr. iur. Friedrich Merzbacher, Würzburg, Staatsarchiv, S. 376.

Prof. Dr. Hermann N ottarp in Würzburg, S. 433, 436, 437, 446, 463.

Prof. Dr. Percy Ernst Schramm in Göttingen, S. 394.

Prof. Dr. Wilhelm Weizsäcker in Heidelberg, S. 396. 